\title{
Dissecting the immune component of neurologic disorders: a grand challenge for the 21st century
}

\section{Patricia Katherine Coyle* \\ Department of Neurology, Stony Brook University Medical Center, Stony Brook, NY, USA \\ *Correspondence: pcoyle@notes.cc.sunysb.edu}

The Grand Challenge for multiple sclerosis (MS) and Neuroimmunology in the 21 st century is to decipher the precise role played by the host immune system in MS and other neurologic diseases, and then use that knowledge to design effective immunemediated therapeutic strategies. This will be a very tall order, because in essence it means dissecting etiologies, and establishing treatments for, a host of common and rare nervous system diseases. It is not out of the realm of possibility however, given the enormous strides being made in understanding how the nervous and immune systems interact.

The immune system is a complex mobile and reactive entity that performs surveillance on, and comes in contact with, every organ of the body. It can be modified and change over time. Knowledge about immune system components and characteristics is constantly evolving. Recent focus has been on understanding molecular communications, systems networks, and pathogen recognition receptors, and how their dysregulation can cause immune disturbances. Many of these concepts were unfamiliar just a few short years ago. Immunology taught during medical and graduate school seems to become quickly outdated. As we learn more, the list of neurologic diseases in which the immune system plays an important role continues to grow. In light of the critical ties between the nervous and immune systems, every neurologist should understand current neuroimmunologic principles.

Disorders under the umbrella of Neuroimmunology are not just the prototypic immune-mediated central (CNS) and peripheral nervous system (PNS) disorders, such as MS and Myasthenia Gravis. There are also a host of novel syndromes. One example is Hashimoto's encephalopathy (Mocellin et al., 2007). This disorder, associated with normal thyroid function but very high antithyroid antibody titers, presents with dramatic neuropsychiatric and cognitive abnormalities, along with focal deficits, movement disorders, and seizures. It is important to recognize, since it is a corticosteroid-responsive encephalopathy. Another example involves a spectrum of disorders resulting from immune reactivity to synapse components. They produce a wide variety of neuropsychiatric disturbances, characterized by catatonia, memory deficits, movement disorders, psychosis, and seizures (Rosenfeld and Dalmau, 2011). These disorders can affect children and young adults, in addition to older individuals. Unrecognized and untreated, these are devastating illnesses. With appropriate immunotherapy, even a moribund patient can make excellent recovery. Paraneoplastic disorders are yet another expanding neuroimmune area (Greenlee, 2010). They are defined as remote effects of malignancies, and can target virtually any site within the neuraxis (CNS, PNS, or neuromuscular junction). Typically there is a host immune response against one or more intracellular or cell membrane neuronal or glial antigens, often marked by suggestive antibodies. These syndromes can present well before the neoplasm has declared itself. In most cases the paraneoplastic syndrome is so characteristic that its recognition should trigger a tumor search. Therapy involves treating the associated malignancy, along with institution of immunotherapy. It is worth noting that virtually all major neurologic conditions (including Alzheimer disease, cerebrovascular disease, epilepsy, Parkinson's, and CNS infection) are now recognized to have immune/inflammatory components. In fact, immunologic therapeutic strategies (such as anti-inflammatory agents, intravenous immunoglobulins, monoclonal antibodies, and vaccinations) are being tested in many of these disorders. This would have been unthinkable several years ago. It seems there is hardly a neurologic disease where Neuroimmunology is not involved. Recent studies even indicate that neuroimmune interactions control the generation of new functional neurons from neural stem cells (Molina-Holgado and Molina-Holgado,
2010). This puts neuroimmune interventions front and center when designing CNS repair strategies.

Multiple sclerosis remains the classic example of a CNS neuroimmune disorder. In the past few years knowledge about MS has advanced, with remarkable new insights. Yet these advances have also produced new questions and issues. MS is clearly not an inherited disease, although genetics play a key role and MS is recognized to be polygenic. The linked genes preferentially involve immune responses, cell adhesion, cell communications and signaling, and nervous system development (Wang et al., 2011). Ongoing studies are aimed at identifying genes that facilitate development of MS, protect against it, and control disease severity. However, environmental factors appear to trump genetics. The implicated factors include vitamin D deficiency, clinical Epstein Barr virus infection (mononucleosis), tobacco use, lack of exposure to pathogens in early life, and most recently solar radiation (Ortor et al., 2011). How they influence development of MS needs to be determined.

Multiple sclerosis is now recognized to be heterogenous. It does not simply reflect $\mathrm{T}$ cells attacking myelin. B cells, chemokines, microglia, oxygen free radicals, and glutamate are also implicated. MS is much more than just a demyelinating, white matter disease. Gray matter is involved early. There is neurodegeneration, with loss of axons and neurons, that seems to underlie the slow worsening progressive clinical subtype. Targets of attack have expanded beyond myelin and axon. The most recent topics of interest focus on glial cells; CNS ion channels, ion exchanges, and ion transporters; mitochondria; and blood vessels, including the venous system.

One of the most interesting areas of advancement in MS has been the delineation of distinct neuroimmune disorders originally considered part of the MS spectrum: acute disseminated encephalomyelitis 
(ADEM), and neuromyelitis optica (NMO)Devic disease. ADEM is a postinfectious or postvaccine encephalomyelitis that typically affects children, is monophasic, and produces a clinical syndrome of encephalopathy accompanied by focal neurologic deficits. It is distinct from MS not just on clinical grounds, but also on neuroimaging, cerebrospinal fluid, and pathologic abnormalities, as well as etiology. Children with ADEM are at risk to go on to develop MS, in contrast to adults.

Not that long ago, NMO-Devic disease was also considered a variant of MS. It is now recognized that most patients have a unique neuroimmune disorder that reflects an immune attack against an astrocyte target, aquaporin 4 (Fujihara, 2011; Idiman and Ozalbas, 2011). Aquaporin 4 is a water channel component on the astrocyte foot process, and plays an important role in maintaining the blood brain barrier. IgG antibodies to aquaporin 4 have become an important diagnostic and prognostic biomarker. NMO-Devic disease also shows distinct etiology, pathology, neuroimaging, and CSF profiles from MS. It has a different prognosis, and responds to different therapeutic approaches. That is why it is so crucial to differentiate NMO-Devic disease from MS. Study of NMO-Devic disease is producing new insights applicable to neuroimmune disorder, including MS. It has identified a new antigen target responsible for producing it, and a sensitive diagnostic biomarker antibody. It proves that astrocyte dysfunction can produce an inflammatory demyelinating CNS process. This provides a model to rethink MS. Could MS reflect a primary astrocyte disturbance? In addition, NMO-Devic disease has been linked to
Sjogren disease, a rheumatologic connective tissue disorder. The linkage likely reflects the fact that aquaporin 5 is a major component of salivary gland, and shows $50 \%$ homology with aquaporin 4 . Sjogren disease itself can involve the CNS, and is in the differential diagnosis of MS. Perhaps this aquaporin link will prove key to explaining its CNS involvement.

For MS the Grand Challenge will be to understand disease etiology better (identify critical genes, environmental factors, heterogeneous damage mechanisms). This will allow as to develop an effective strategy to treat the neurodegenerative progressive phase of the disease. Right now this is a major gap, since the approved disease modifying therapies have only been shown to benefit relapsing forms of MS. MS awaits identification of meaningful biomarkers to guide treatment decisions. Finally, effective CNS repair strategies are needed. This will provide hope and assistance to patients with fixed deficits.

The Grand Challenge for Neuroimmunology in general will be to continue to identify those neurologic disorders in which the immune system plays a key role, and to develop effective therapeutic strategies accordingly. Scientific advances are leading to amazing developments. Optogenetics, using viral vectors to insert light-sensitive channels in neurons, allows targeted neurons to be turned on or off based on a simple light stimulus. Neural stimulation, depending on the CNS site, is being proposed to treat cognitive loss, depression, inability to walk, and obsessive compulsive disorder. It is exciting to look forward to understanding, then controlling, the role of the immune system in neurologic disease.

\section{REFERENCES}

Fujihara, K. (2011). Neuromyelitis optica and astrocytic damage in its pathogenesis. J. Neurol. Sci. [Epub ahead of print].

Greenlee, J. E. (2010). Treatment of paraneoplastic neurologic disorders. Curr. Treat. Options Neurol. 12, 212-230.

Idiman, E., and Ozakbas, S. (2011). The limited demyelinating diseases: the voyage of optic neuritis and transverse myelitis to multiple sclerosis and neuromyelitis. Expert Rev. Neurother. 11, 451-462.

Mocellin, R., Walterfang, M., and Velakoulis, D. (2007). Hashimoto's encephalopathy: epidemiology, pathogenesis and management. CNS Drugs 21, 799-811.

Molina-Holgado, E., and Molina-Holgado, F. (2010). Mending the broken brain: neuroimmune interactions in neurogenesis. J. Neurochem. 114, 1277-1290.

Ortor, S. M., Wald, L. Confavreux, C., Vukusic S, Krohn, J.P., Ramagopalan, S. V., Herrera, B. M., Sadovnick, A. D., and Ebers, G. C. (2011). Association of UV radiation with multiple sclerosis prevalence and sex ratio in France. Neurology 76, 425-431.

Rosenfeld M. R., and Dalmau J. (2011). Anti-NMDAreceptor encephalitis and other synaptic autoimmune disorders. Curr. Treat. Options Neurol. 13, 324-332.

Wang, J. H., Pappas, D., De Jager, P. K., Pelletier, D., de Bakker, P. I., Kappos, L., Polman, C. H., Australian and New Zealand Multiple Sclerosis Genetics Consortium (ANZgene), Chibnik, L. B., Hafler, D. A., Matthews, P. M., Hauser, S. L., Baranzini, S. E., and Oksenberg, J.R. (2011). Modeling the cumulative genetic risk for multiple sclerosis from genome wide association data. Genome Med. 3, 3 .

Received: 16 March 2011; accepted: 19May 2011;published online: 06 June 2011.

Citation: Coyle PK (2011) Dissecting the immune component of neurologic disorders: a grand challenge for the 21st century. Front. Neur. 2:37. doi: 10.3389/fneur.2011.00037 This article was submitted to Frontiers in Multiple Sclerosis and Neuroimmunology, a specialty of Frontiers in Neurology.

Copyright () 2011 Coyle. This is an open-access article subject to a non-exclusive license between the authors and Frontiers Media SA, which permits use, distribution and reproduction in other forums, provided the original authors and source are credited and other Frontiers conditions are complied with. 